\title{
Squeezed Light for the Interferometric Detection of High Frequency Gravitational Waves
}

\author{
R. Schnabel $\nmid \S$, J. Harms $\dagger$, K. A. Strain $\ddagger$, and K. Danzmann $\dagger$ \\ $\dagger$ Max-Planck-Institut für Gravitationsphysik (Albert-Einstein-Institut) and \\ Institut für Atom- und Molekülphysik, Universität Hannover, 30167 Hannover, Germany \\ $\ddagger$ Department of Physics and Astronomy, University of Glasgow, Glasgow G12 8QQ, UK
}

\begin{abstract}
The quantum noise of the light field is a fundamental noise source in interferometric gravitational wave detectors. Injected squeezed light is capable of reducing the quantum noise contribution to the detector noise floor to values that surpass the so-called Standard-Quantum-Limit (SQL). In particular, squeezed light is useful for the detection of gravitational waves at high frequencies where interferometers are typically shot-noise limited, although the SQL might not be beaten in this case. We theoretically analyze the quantum noise of the signal-recycled laser interferometric gravitational-wave detector GEO 600 with additional input and output optics, namely frequency-dependent squeezing of the vacuum state of light entering the dark port and frequency-dependent homodyne detection. We focus on the frequency range between $1 \mathrm{kHz}$ and $10 \mathrm{kHz}$, where, although signal recycled, the detector is still shot-noise limited. It is found that the GEO 600 detector with present design parameters will benefit from frequency dependent squeezed light. Assuming a squeezing strength of $-6 \mathrm{~dB}$ in quantum noise variance, the interferometer will become thermal noise limited up to $4 \mathrm{kHz}$ without further reduction of bandwidth. At higher frequencies the linear noise spectral density of GEO 600 will still be dominated by shot-noise and improved by a factor of $10^{6 \mathrm{~dB} / 20 \mathrm{~dB}} \approx 2$ according to the squeezing strength assumed. The interferometer might reach a strain sensitivity of $6 \times 10^{-23}$ above $1 \mathrm{kHz}$ (tunable) with a bandwidth of around $350 \mathrm{~Hz}$. We propose a scheme to implement the desired frequency dependent squeezing by introducing an additional optical component to GEO 600s signal-recycling cavity.
\end{abstract}

PACS numbers: 04.80.Nn, 03.65.Ta, 42.50.Dv, 95.55.Ym

\section{Introduction}

It was first proposed by Caves [1] that squeezed light injected into the dark port of a gravitational wave $(\mathrm{GW})$ interferometer can be employed to reduce the high laser power requirements. Later Unruh [2] and others [3, 4, 5, 6] have found and proven in different ways that squeezed light with a frequency dependent orientation of the squeezing ellipse can reduce the quantum noise over the complete spectrum. Therefore quantum noise spectral densities below the so-called standard quantum limit (SQL) are possible. In all cases interferometer topologies without signal-recycling were considered. Chickarmane et al. [7, 8] investigated the signal-recycled interferometer with Fabry-Perot arm cavities and injected squeezed light. Radiation pressure noise was not taken into account. Recently Harms et al. [9] have shown that high power signal-recycled interferometers will benefit from squeezed light similarly to conventional interferometers.

GEO 600 [10] is the only first-generation detector that not only uses power recycling [11], but also includes the more advanced technique of signal recycling. The original idea

$\S$ To whom correspondence should be addressed (Roman.Schnabel@aei.mpg.de) 


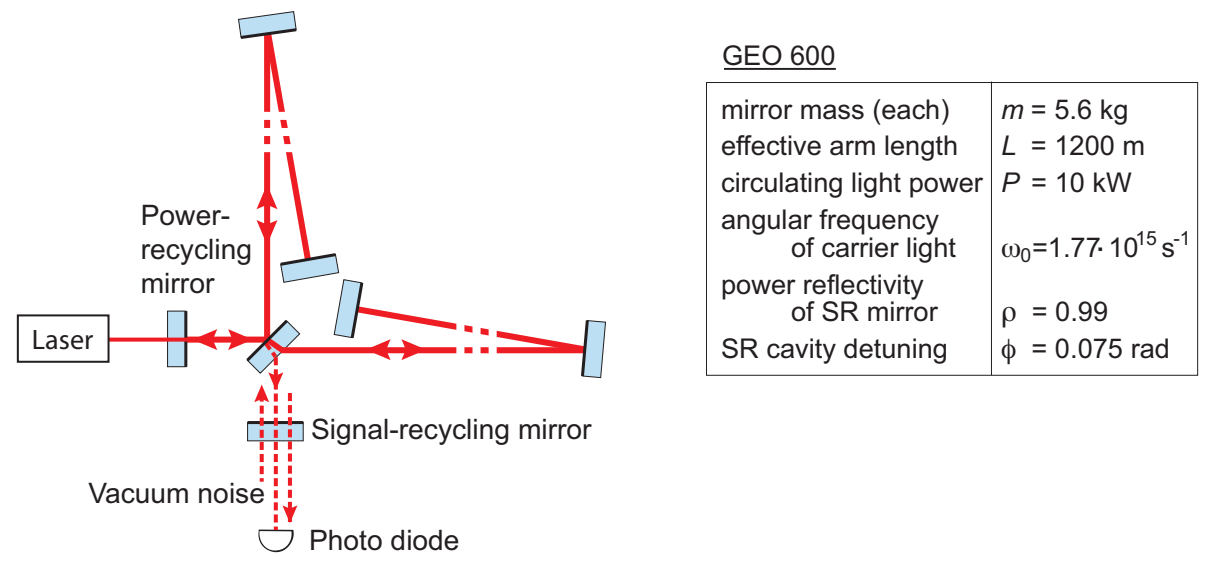

Figure 1. GEO 600 is a dual-recycled Michelson interferometer with a power-recycling mirror in the bright port that enhances the light power within the Michelson arms and a signalrecycling mirror in the dark port that can be tuned on a specific signal frequency. Since the arms are folded once, then the effective arm length is doubled to $1200 \mathrm{~m}$. Vacuum noise enters the interferometer from the dark port and is back reflected onto the photo diode. The table provides technical data and parameter values of GEO 600 which where used to calculate the noise spectral densities in Figs. 2 and 4a; SR: signal-recycling.

of the signal-recycling (SR) was due to Meers [12], who proposed its use for dual-recycling, which is the combination of power- and signal-recycling. With signal recycling, part of the GW induced signal light is retro-reflected at the dark port and back into the interferometer, establishing an additional cavity which can be set to resonate at a desired gravitational-wave frequency (Fig. 1). Signal recycling leads to a well known (optical) resonance structure in the interferometer's noise curve. This resonance can already beat the standard quantum limit even in the absence of squeezed light input [13]. Buonanno and Chen have also predicted a second, opto-mechanical resonance in signal-recycled interferometers, around which the interferometer gains sensitivity, and which can also beat the standard quantum limit $[13,14,15,16]$.

In this paper, we investigate the benefit of injecting squeezed vacuum with frequencydependent squeezing angle into GEO600s dark port. We focus on the frequency range between $1 \mathrm{kHz}$ and $10 \mathrm{kHz}$ where the interferometer is expected to be shot-noise limited. We use the two-photon input-output formalism of quantum optics [18] which has also been used by Harms et al. in [9]. Our results are presented in terms of noise spectral densities and are compared with the thermal noise expected for GEO 600. Several different types of astrophysical sources have been predicted, that might radiate gravitational waves in the $\mathrm{kHz}$ spectrum. Generally speaking, small objects of 1 to 10 solar masses and of about $10 \mathrm{~km}$ diameters are considered. Due to theoretical models, neutron star bar-mode instabilities, black hole ringing, merging neutron star binaries [17] and other sources are discussed. For an overview we refer to the article by Kokkotas in this issue.

\section{Spectral noise densities of GEO 600}

GEO 600 is, as already mentioned above, a dual-recycled interferometer with folded interferometer arms. Recently the GEO 600 interferometer in Ruthe near Hannover has been completed by the implementation of the signal-recycling mirror. Relevant technical 
parameters of GEO 600 are summarized in Fig. 1.

Here we are interested in the optical noise of GEO 600 above $1 \mathrm{kHz}$. Quantum noise and thermal noise have to be considered. Generally, the optical noise in an interferometer can be expressed in terms of the (single-sided) noise spectral density of the output field normalized by the transfer function of the signal. Fig. 2a shows expected linear noise spectral densities (square root of the noise spectral densities) of the GEO 600 interferometer. The upper grey curved line in Fig. 2a represents the quantum noise in the amplitude quadrature. The straight solid lines represents the internal $(\propto \sqrt{1 / f})$ and the photo-refractive thermal noise limits $(\propto 1 / f)$. GEO 600s standard quantum limit (SQL) is given as a reference (straight dashed line):

$$
\sqrt{h_{\mathrm{SQL}}}=\sqrt{\frac{20 \hbar}{m \Omega^{2} L^{2}}} .
$$

We have used the two-photon input-output relation of quantum optics [18], which maps the numerous input fields $\overline{\mathbf{i}}_{n}$ and the gravitational-wave signal $h=\Delta L / L$ onto the detected output field $\overline{\mathbf{o}}$. $\overline{\mathbf{i}}$ and $\overline{\mathbf{o}}$ are two-dimensional vectors, where the first components represent the fields amplitude quadrature and the second ones the phase quadrature. The general expression for the resulting quantum noise spectral density $S_{h}$ can be found in [9]. We note that optical losses and radiation pressure noise at the beam-splitter is not considered here. According to the table in Fig. 1 we chose the signal-recycling cavity to be detuned from the carrier frequency by $0.075 \mathrm{rad}$. The SR cavity is therefore resonant for $3 \mathrm{kHz}$ gravitational wave signals. It can be seen in Fig. 2a that at this optical resonance GEO 600 is expected to be quantum noise (shot-noise) limited. The second resonance at lower frequencies stems from the classical opto-mechanical coupling of the light field with the anti-symmetric mode of the otherwise free mirrors [15]: in detuned signal-recycling schemes, the phase-modulation sidebands induced by a gravitational wave are partly converted into amplitude modulation, which beat with the carrier field, producing a motion-dependent force and acting back on the test masses. This opto-mechanical resonance is likely to be buried by thermal noise which is also expected for GEO 600. Only above $1 \mathrm{kHz}$ will squeezed light injected into the dark port of the interferometer be able to reduce the overall noise-floor.

For all noise spectra in this paper we assume homodyne detection at the dark port of the interferometer. The detected quadrature of angle $\zeta$ is assumed to be the amplitude quadrature in respect to the carrier field inside the interferometer $(\zeta=0)$. An appropriate local oscillator can be provided by differential loss in the two interferometer arms. In the case of no differential loss, but while locking the interferometer slightly offset to the dark fringe, the phase quadrature will be detected which is the upper dashed line in Fig. 4a. Apart from more quantum noise at intermediate frequencies, all results from this paper remain valid for homodyne detection of the phase quadrature or of any other quadrature of angle $\zeta$. The quantum noise in a heterodyne readout scheme has also been analyzed and tends towards higher noise floors [19].

\section{Squeezed light enhanced GEO 600}

Generally, quantum noise can be understood in terms of vacuum fluctuations. Since the interferometer is operated at the dark fringe, vacuum fluctuations impinging on the photo diode at the interferometers dark port stem from vacuum fluctuations that have been reflected back. Indeed the beat between these vacuum fluctuations and the carrier laser light entering the bright port excites the interferometers anti-symmetric mode of motion. On the other hand, vacuum noise entering the bright port excites the irrelevant symmetric mode of motion 

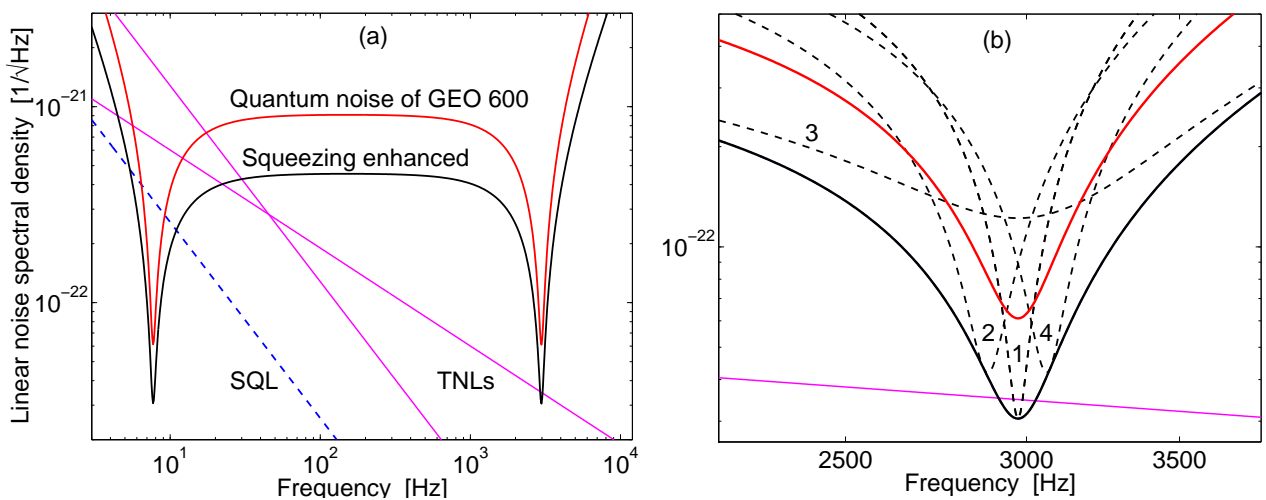

Figure 2. a) The linear quantum noise spectral density can be reduced by a factor of $10^{x / 20 \mathrm{~dB}}=\mathrm{e}^{-\mathrm{r}}$, where $\mathrm{x}$ is the relative squeezing of the variance and $\mathrm{r}$ is the so-called squeezing parameter. Here we chose $x=6 \mathrm{~dB}$. The amplitude quadrature is shown with and without optimally frequency dependent squeezed light again in comparison with the standard quantum limit (SQL) and thermal noise limits (TNLs).

b) Zoom into the optical resonance. The dashed lines give the results of applying frequency independent squeezed light, where the squeezing is chosen to be in amplitude (curve 1), at $45^{\circ}$ (2), in phase (3) and at $-45^{\circ}$ (4). The lower curve is again the optimum achievable, when applying the correct squeezing angle at every frequency.

and does not effect the quantum noise of the GW detector. For this reason, squeezed light is planned to be injected into the interferometers dark port. Recently, a scheme using a Faraday rotator has been experimentally demonstrated on a table-top power-recycled Michelson-interferometer [20]. Squeezed light is currently most efficiently generated in optical parametric amplifiers (OPAs). At a laser wavelength of $1064 \mathrm{~nm}, \mathrm{MgO}: \mathrm{LiNbO}_{3}$ crystals are heated to about $107^{\circ} \mathrm{C}$ to meet the phase matching condition between the fundamental and the green second harmonic pump frequency. The relative phase shift of both frequencies can be locked by a servo loop and determines what quadrature of the infrared OPA output will be squeezed. The orthogonal quadrature will then be anti-squeezed at least by the same factor due to the Heisenberg uncertainty principle. A reduction of quantum noise variances of up to $7 \mathrm{~dB}$ has been reported at sideband frequencies of a few $\mathrm{MHz}$ with squeezing bandwidths of also a few $\mathrm{MHz}$ [21], [22]. For the use in GW interferometers broadband squeezed light at $\mathrm{GW}$ frequencies needs to be generated with a frequency dependent rotation of the squeezed quadrature. The lowest frequency that squeezed light has been demonstrated at so far is at $220 \mathrm{kHz}$ [23]. Frequency dependent squeezing has not been demonstrated yet.

Fig. 2a shows the reduction of quantum noise in the amplitude quadrature expected for GEO 600 if a non-classical vacuum state which is optimally frequency dependently squeezed in variance by $-6 \mathrm{~dB}$ throughout the complete spectrum from 3 to $10000 \mathrm{~Hz}$ is injected into the dark port. In Fig. $2 b$ we zoom into the optical resonance. The dashed lines give the results of applying frequency independent squeezed light, where the squeezing is chosen to be in amplitude, at $45^{\circ}$, in phase and at $-45^{\circ}$, respectively. It is well-known that frequency dependent squeezed light is crucial for a $\mathrm{GW}$ interferometer to benefit from non-classical quantum noise reduction. We therefore focus now on the issue how to implement appropriate filter cavities that convert frequency independent into frequency dependent squeezed vacuum states of light.

As said above, squeezed vacuum can be generated with a variable but frequency- 

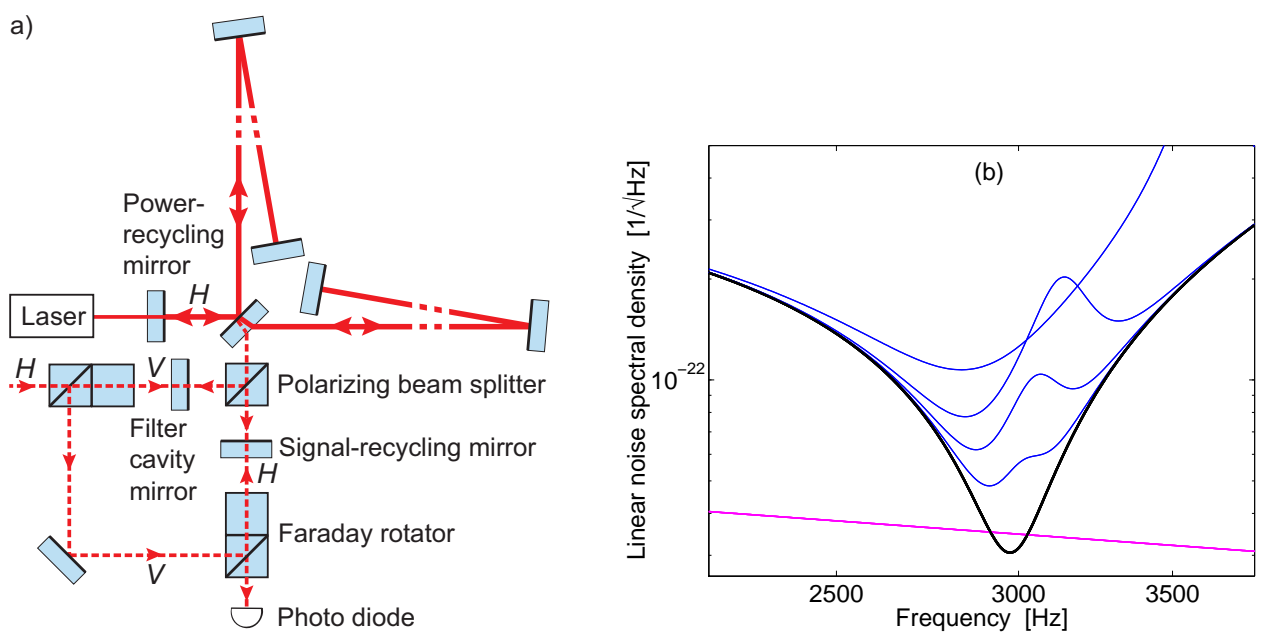

Figure 3. a) Proposed scheme to cancel the phase shift on frequency independent squeezed light. $H$ and $V$ label the horizontal and vertical polarization modes respectively.

b) The detuning of the filter cavity is varied from its here optimum value of $\phi_{\mathrm{FP}}=$ $-0.075 \mathrm{rad}$ by $-0.016,-0.004,-0.002$ and $-0.001 \mathrm{rad}$ (from top to bottom).

independent squeezing angle $\lambda$ (see for example [24]). A frequency-dependent squeezing angle can then be obtained by subsequently filtering the initial squeezed light through detuned Fabry-Perot (FP) cavities, as proposed by Kimble et al. [6] for so-called quantum-nondemolition interferometers. Harms et al. found that for signal-recycled GW interferometers, two filter cavities are necessary to cancel rotations due to the SR cavity as well as rotation due to the radiation pressure force if the readout quadrature is frequency independent [9]. Here we consider an optical resonance which is well separated from the opto-mechanical one. Therefore the radiation pressure noise need not to be taken into account and the quantum noise in Fig. $2 \mathrm{a}$ above $1 \mathrm{kHz}$ is solely due to shot noise. The rotation due to the SR cavity is therefore the only one which needs to be cancelled, again if the readout quadrature is frequency independent. As an example, an appropriate single filter cavity, labeled by FP, could match the SR cavity parameters apart from a negative detuning from the carrier light $\phi$ :

$$
\Omega^{\text {filter res }}=\frac{\phi_{\mathrm{FP}} c}{L_{\mathrm{FP}}}-\mathrm{i} \frac{\mathrm{c} \tau_{\mathrm{FP}}^{2}}{4 \mathrm{~L}_{\mathrm{FP}}}=-\frac{\phi \mathrm{c}}{\mathrm{L}}-\mathrm{i} \frac{\mathrm{c} \tau^{2}}{4 \mathrm{~L}} .
$$

Here $\Omega^{\text {filter res }}$ is the filter cavity resonant frequency, $L$ is the cavity length and $\tau$ the transmissivity of the cavity input mirror. When detecting the quadrature at angle $\zeta$, the required initial (frequency independent) squeezing angle $\lambda_{0}$ is found to be

$$
\lambda_{0}=\zeta-\pi / 2,
$$

which puts the minor axis of the noise ellipse onto the $\zeta$ quadrature. Note, here $\lambda_{0}=0$ defines squeezing of the phase quadrature, according to definitions in Eq. (12) of Ref. [9]. If for example the amplitude quadrature of the light field at the dark port is detected, then, at the SR cavity resonant frequency, the amplitude quadrature needs to be squeezed. The phase reference is set by the carrier light field inside the Michelson interferometer. For a more general consideration in case of more filter cavities required we refer to the work of Purdue and Chen in Appendix A of Ref. [25] and to the work of Harms et al. [9].

In Fig. 3a we propose a scheme that implements the desired single filter cavity. Lets assume that the laser light inside the interferometer is horizontally polarized. A polarizing 
beam splitter is placed between the SR mirror and the interferometers beam splitter. A mirror matching the SR mirror is added to form an additional cavity, now for the vertical mode. This new cavity can be detuned independently from the SR cavity and can thereby provide the desired filter cavity. If $\phi_{\mathrm{FP}}=-\phi$ then the phase shift on squeezed vacuum noise that is subsequently reflected from both cavities cancels to zero. This is shown in Fig. $3 \mathrm{~b}$ where the detuning of the filter cavity is varied at the fixed detuning of the SR cavity.

\section{Variational output}

The quantum noise spectral density of interferometers can benefit simultaneously and independently from both frequency-dependent squeezed light input, and frequencydependent homodyne read-out (variational output). This has been shown for conventional interferometers without signal-recycling by Kimble et al. [6] and for optical-spring signalrecycled interferometers by Harms et al. [9]. Both the squeezed input and the variational output schemes require additional optics and rotating filter cavities.
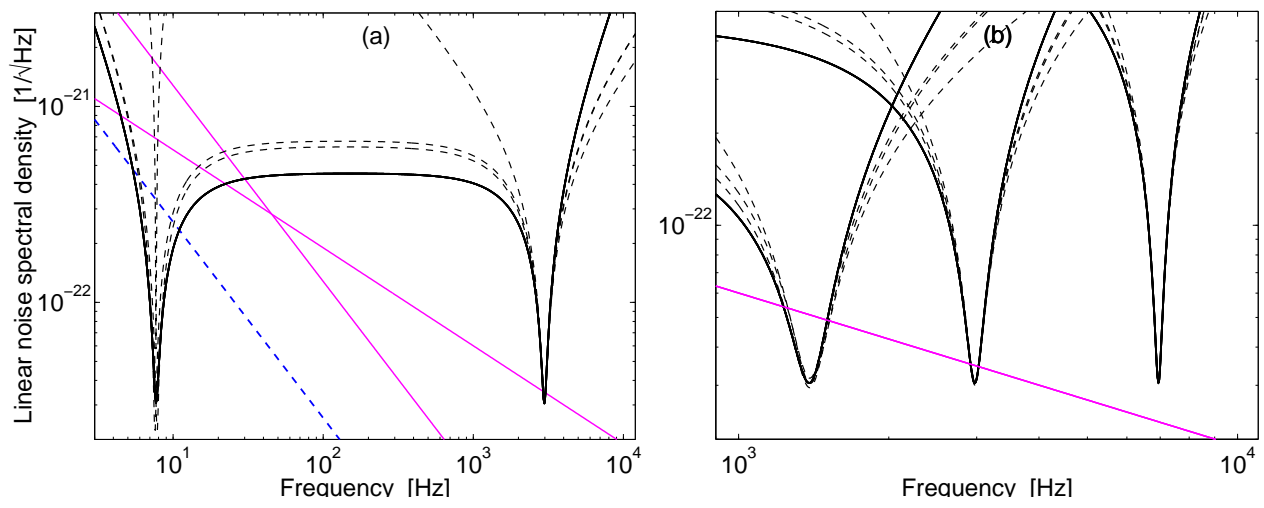

Figure 4. a) The curved lines represent squeezed light improved quantum noise spectral densities for four different quadrature angles.

b) Zoom into the optical resonance, now for three different SR cavity detunings.

Starting from the squeezing enhanced curve in Fig. 2a, we have plotted three additional lines (dashed) in Fig. 4a. Together with the lower solid curve they form an array of squeezed spectral noise densities at different fixed output quadrature detection angles $\zeta$. Here $\zeta$ is varied from 0 (solid line) to $3 / 4 \pi$ in equidistant steps. It can be seen that above the optical resonance and at the opto-mechanical resonance non-zero values for $\zeta$ provide lower quantum noise floors. The lower boundary of the array is indeed achievable by applying the variational output scheme. In Fig. 4b we zoom into the optical resonance and consider three different detunings of the SR-cavity ( $\phi=0.035,0.075$ and $0.175 \mathrm{rad})$. It can be seen that at all three resonance frequencies, variational output will give an improvement, but only far above the noise minima in the wings of the resonances. This improvement is not significant for GEO 600s noise-floor above $1 \mathrm{kHz}$.

\section{Conclusion}

We have shown that squeezed light will be able to reduce the overall noise floor of the signal-recycled gravitational wave interferometer GEO 600 at frequencies above $1 \mathrm{kHz}$, where 
GEO 600 is expected to be shot-noise limited. Our investigations are based on a non-classical vacuum state of light with quantum noise variance reduced by $-6 \mathrm{~dB}$ at sideband frequencies between 1 and $10 \mathrm{kHz}$. If such a state is injected into the dark port of the interferometer, then GEO 600 with its current design parameters can be made thermal noise limited up to $4 \mathrm{kHz}$ without further reduction of bandwidth. The interferometer might reach a strain sensitivity of $6 \times 10^{-23}$ above $1 \mathrm{kHz}$ (tunable) with a bandwidth of around $350 \mathrm{~Hz}$. An optimized rotation of the squeezed quadrature along the spectrum is one of the essential properties of the squeezed light used. We have shown, for the special case considered here, that just one filter cavity is sufficient to provide the desired rotation, and can be realized from GEO 600s signal-recycling cavity with additional polarizing optics.

[1] C. M. Caves, Phys. Rev. D 23, 1693 (1981).

[2] W. G. Unruh, in Quantum Optics, Experimental Gravitationa, and Measurement Theory, edited by P. Meystre and M. O. Scully (Plenum, New York, 1982), p. 647.

[3] J. Geabanacloche and G. Leuchs, J. Mod. Opt. 34, 793 (1987).

[4] M. T. Jaekel and S. Reynaud, Europhys. Lett. 13, 301 (1990).

[5] A. F. Pace, M. J. Collett, and D. F. Walls, Phys. Rev. A 47, 3173 (1993).

[6] H. J. Kimble, Y. Levin, A. B. Matsko, K. S. Thorne, and S. P. Vyatchanin, Phys. Rev. D 65, 022002 (2001).

[7] V. Chickarmane and S. V. Dhurandhar, Phys. Rev. A 54, 786 (1996).

[8] V. Chickarmane, S. V. Dhurandhar, T. C. Ralph, M. Gray, H.-A. Bachor, and D. E. McClelland, Phys. Rev. A 57, 3898 (1998)

[9] J. Harms, Y. Chen, S. Chelkowski, A. Franzen, H. Vahlbruch, K. Danzmann, and R. Schnabel, Phys. Rev. D 68, 042001 (2003).

[10] B. Willke et al., Class. Quantum Grav. 19, 1377 (2002).

[11] R. W. P. Drever et al. in Quantum Optics, Experimental Gravitationa, and Measurement Theory, edited by P. Meystre and M. O. Scully (Plenum, New York, 1983), p. 503-514.

[12] B. J. Meers, Phys. Rev. D 38, 2317 (1988).

[13] A. Buonanno and Y. Chen, Phys. Rev. D 64, 042006 (2001).

[14] A. Buonanno and Y. Chen, Class. Quantum Grav. 18, L95 (2001).

[15] A. Buonanno and Y. Chen, Phys. Rev. D 65, 042001 (2002).

[16] A. Buonanno and Y. Chen, Phys.Rev. D 67, 062002 (2003).

[17] C. L. Fryer, D. E. Holz, and S. A. Hughes, Astrophys. J. 565, 430 (2002).

[18] C. M. Caves and B. L. Schumaker, Phys. Rev. A 31, 3068 (1985); B. L. Schumaker, and C. M. Caves, Phys. Rev. A 31, 3093 (1985).

[19] A. Buonanno, Y. Chen and N. Mavalvala, Phys. Rev. D 67, 122005 (2003).

[20] K. McKenzie, D. A. Shaddock, D. E. McClelland, B. C. Buchler, and P. K. Lam, Phys. Rev. Lett. 88, 231102 (2002).

[21] K. Schneider, M. Lang, J. Mlynek, and S. Schiller, Opt. Ex. 2, 59 (1998).

[22] P. K. Lam, T. C. Ralph, B. C. Buchler, D. E. McClelland, H.-A. Bachor, and J. Gao, J. Opt. B 1, 469 (1999).

[23] W. P. Bowen, R. Schnabel, N. Treps, H.-A. Bachor, and P. K. Lam, J. Opt. B, 4, 421 (2002).

[24] G. Breitenbach, F. Illuminati, S. Schiller, and J. Mlynek, Europhys. Lett. 44, 192 (1998).

[25] P. Purdue and Y. Chen, Phys. Rev. D 66122004 (2002). 